\title{
Aprendizaje y práctica de la conciencia plena en estudiantes de bachillerato para potenciar la relajación y la autoeficacia en el rendimiento escolar*
}

\author{
Learning Mindfulness for Enhancing Relaxation and Self- \\ Efficacy on Academic Performance in High School Students
}

Recibido: 21 de junio de 2013 | Revisado: 17 de junio de 2014 | Aceptado: 5 de febrero de 2015

\author{
Alberto Amutio-KareagA ** \\ Universidad del País Vasco, Vizcaya, España \\ Clemente Franco Justo *** \\ JOSÉ JESÚS GÁZQUEZ LINARES ***** \\ ISRAEL MAÑAS MAÑAS****** \\ Universidad de Almería, Almería, España
}

doi:10.11144/Javeriana.upsy14-2.apcp

Para citar este artículo: Amutio-Kareaga, A., Franco, C., Gázquez, J. J., \& Mañas, I. (2015). Aprendizaje y práctica de la conciencia plena en estudiantes de bachillerato para potenciar la relajación y la autoeficacia en el rendimiento escolar. Universitas Psychologica, 14(2), 433-444. http:// dx.doi.org.10.11144/Javeriana.upsy14-2.apcp

* Artículo de investigación

*** Profesor Agregado de Universidad. Departamento de Psicología Social y Metodología de las Ciencias del Comportamiento. Universidad del País Vasco (UPV/EHU). Avda Tolosa, 70. Donostia-San Sebastián. 20018. Teléfono: 943015674. Correo electrónico: alberto.amutio@ehu.es

**** Profesor Titular de Universidad. Departamento de Psicología. Universidad de Almería. La Cañada de San Urbano s/n. Almería. 04120. Teléfono: 950015499. Correo electrónico: cfranco@ual.es

***** Profesor Titular de Universidad. Departamento de Psicología. Universidad de Almería. La Cañada de San Urbano s/n. Almería. 04120. Teléfono: 950015598.Correo electrónico: jlinares@ual.es

${ }^{* * * * * * *}$ Profesor Sustituto Interino. Departamento de Psicología. Universidad de Almería. La Cañada de San Urbano s/n. Almería. 04120. Teléfono: 950015819. Correo electrónico: imanas@ual.es
RESUMEN

El objetivo este estudio fue analizar los efectos que produce un programa psicoeducativo de entrenamiento en conciencia plena (mindfulness) sobre la autoeficacia en el rendimiento escolar y los estados de relajación (Estados$\mathrm{R}$ ), en adolescentes estudiantes de bachillerato. Se realizó un diseño controlado y aleatorizado con un grupo experimental y un grupo control en lista de espera con medición pretest-postest. Los instrumentos de medida empleados fueron el Cuestionario de Cartagena (Cartagena, 2008) y el Cuestionario SRSI-3 (Smith, 2005a). Los análisis estadísticos muestran la existencia de mejoras significativas en el grupo experimental en comparación con el grupo control, en los niveles de autoeficacia en el rendimiento escolar, así como en los estados de relajación básica, conciencia plena y energía positiva; además, se obtuvo una reducción significativa en el grupo experimental en comparación con el grupo control en la dimensión de estrés percibido. Palabras clave

conciencia plena; autoeficacia escolar; estados de relajación; estudiantes de bachillerato

\section{A B S T R A C T}

The aim of the present study is to analyze the effects of a mindfulness training psycho-educative program on self-efficacy in the academic performance and relaxation states in a sample of High School students. A randomized controlled trial with pretest-posttest measurements was used with an experimental group and a control group (waiting list). The Cartagena Questionnaire (Cartagena, 2008) and the SRSI Questionnaire (Smith, 2005a) were employed. The statistical analyses carried out on the studied variables showed significant improvements in favor of the experimental group compared with the control group on the levels of self-efficacy in academic performance, as well as on the states of basic relaxation, mindfulness and positive energy. The program also produced a significant reduction in the experimental group compared with the control group in the dimension of perceived stress.

Keywords

Mindfulness; academic self-efficacy; relaxation states; high school students 


\section{Introducción}

Una definición generalizada de mindfulness es 'conciencia del momento presente con aceptación' (Bishop et al., 2004). Implica la observación de los pensamientos y reacciones emocionales que ocurren en cada momento sin identificarse con ellos. Así, y por medio de la práctica continuada, las personas aprenden a concentrarse en la tarea que están realizando en el momento, sin que la mente divague o se distraiga. Dicha práctica trae consigo estados de calma y de serenidad. Esto le proporciona al individuo una nueva perspectiva que facilita la reflexión y el aprendizaje.

El origen del mindfulness, o la conciencia o atención plena, se remonta a técnicas de meditación oriental, como el budismo Zen o la práctica de Vipassana. Puede ser concebido como un rasgo psicológico o como una técnica. En cuanto a la primera concepción, algunos autores (Simón, 2006) hacen referencia al mindfulness como una capacidad humana básica y universal consistente en la facultad de ser conscientes de la experiencia tanto física como mental que tiene lugar en cada momento mediante la práctica de la autoconciencia. De forma complementaria, en los últimos años ha surgido un creciente interés por incorporar técnicas de meditación en la práctica terapéutica. Desde este ángulo, mindfulness puede considerarse como un conjunto de ejercicios de meditación y ha sido incluido en diferentes formatos psicoterapéuticos y psicoeducativos, incluyendo el entrenamiento en relajación y manejo del estrés (Delgado \& Amutio, 2011; Kabat-Zinn 2003; Schonert-Reichl \& Lawlor, 2010).

Practicar mindfulness permite contemplar los pensamientos y las sensaciones que se experimentan como eventos que fluyen de forma continua y que solo deben ser notados y observados siendo conscientes de su carácter transitorio y no-permanente. De esta manera, se rompe el patrón habitual de pensar-sentir-actuar, así como el hábito de juzgar y evaluar los pensamientos tomándolos como algo con entidad propia, pues aprendemos a observarlos sin identificarnos con ellos, ni reaccionar ante su presencia de la forma automática habitual (San- tamaría, Cebolla, Rodríguez, \& Miró, 2006). Así, práctica de la conciencia plena buscaría sentir las cosas tal y como suceden, sin intentar controlarlas ni actuar sobre ellas, por lo cual, en cierta medida, funcionaría de manera similar a como actúan las técnicas de exposición. Esto posibilita que la persona fluya, permitiendo que determinadas actividades (emociones, cambios fisiológicos) que operan de forma autónoma en el organismo, y regulados por el sistema nervioso autónomo, funcionen de acuerdo con sus propios sistemas naturales de autorregulación (Vallejo, 2006).

Además, la práctica del mindfulness genera un proceso de conocimiento y aceptación que provoca un mejor acceso a modelos alternativos de relación con los pensamientos y con los sucesos que acontecen, ya que a través del entrenamiento en observar sin juzgar ni analizar, se aprende a tomar una nueva perspectiva y a desarrollar una capacidad de afrontar de forma eficaz los problemas mediante el empleo de estrategias cognitivas más productivas como la defusión cognitiva (Wells, 2002). Este entrenamiento permite, a su vez, aprender a reconocer las primeras señales de aparición de la respuesta de estrés, de tal forma que, mediante la aplicación de las habilidades de aceptación y ecuanimidad que su práctica desarrolla, hay más posibilidades de ser eficaz en el afrontamiento de la situación estresante (Cebolla \& Miró, 2006; Chiesa \& Serreti, 2009).

Kabat-Zinn (1982) impulsó el uso de la meditación mindfulness como procedimiento para el tratamiento de trastornos psicosomáticos, mediante el desarrollo del programa denominado Mindfulness-Based Stress Reduction (MBSR), orientado al tratamiento del estrés en el ámbito de la medicina conductual. Se trata de un programa educativo, basado en una metodología práctica y experiencial. Actualmente, hay abundantes datos que apoyan el uso del mindfulness no solo en la reducción del estrés y del burnout en diferentes colectivos profesionales y estudiantiles, sino en la consecución de mayores niveles de relajación y bienestar (Amutio, 2013, 2011; Choi, Karremans, \& Barendregt, 2012; Franco, 2009a, 2009b, 2010; Mañas, Sánchez, \& Luciano, 2008; Martín- 
Asuero, Rodríguez, Puyol-Ribera, Berenguera, \& Moix, 2013).

En cuanto al ámbito escolar, diferentes investigadores han demostrado la utilidad de la aplicación de las técnicas de relajación y meditación/mindfulness en el ámbito escolar, con el fin de aprender la relajación y de este modo activar los recursos internos de los estudiantes (Calvo, Betancort, \& Díaz, 2009; Delgado et al., 2010; Franco, 2009b; Kaspereen, 2012; López, 2007; Schonert-Reichl \& Lawlor, 2010). Entre los diferentes estudios realizados, cabe destacar el de Beaucheim, Hutchins y Patterson (2008), donde se aplicó un programa de mindfulness a un grupo de 34 estudiantes con problemas de aprendizaje y bajo rendimiento académico, encontrando que después del entrenamiento dicho grupo obtuvo una mejora significativa de su rendimiento académico, el autoconcepto, un aumento de sus habilidades sociales y una disminución de la ansiedad rasgo. Del mismo modo, León (2008) encontró una relación significativa entre la atención plena ( $\mathrm{min}$ dfulness) y el rendimiento académico en estudiantes de educación secundaria. Estos resultados están en consonancia con los obtenidos por Franco (2009b) y Franco, Mañas, Cangas, \& Gallego (2011).

En cuanto a la autoeficacia, esta se define como "las creencias en las propias capacidades para organizar y ejecutar los cursos de acción requeridos que producirán determinados logros o resultados" (Bandura, 1997, p. 3). La autoeficacia proporciona a la persona un mecanismo automotivador que facilita el logro académico. Las creencias de eficacia y la autoobservación de las propias competencias determinan el esfuerzo y la persistencia empleada por la personas para superar los obstáculos y experiencias difíciles. De forma paralela, los estados de ánimo y emociones positivas potencian la autoeficacia y con ella el bienestar y el rendimiento.

En este sentido, se ha demostrado que las técnicas de mindfulness producen efectos beneficiosos sobre las emociones positivas y el bienestar. A nivel biológico, se han registrado cambios bioquímicos en el cerebro asociados a emociones más positivas. Así, por ejemplo, se ha observado cierto incremento en la actividad del lóbulo frontal izquierdo, área donde se gestan y almacenan las emociones positivas.
También se ha comprobado que las personas que emplean más la zona izquierda del cerebro, tardan menos tiempo en eliminar las emociones negativas y la tensión, al tiempo que se produce una reducción de emociones como la ira y la ansiedad (BrefczynskiLewis, Lutz, Schaefer, Levinson, \& Davidson, 2007; Davidson et al., 2003; Vago \& Silbersweig, 2012).

En la actualidad, no existen estudios controlados donde se mida el impacto del entrenamiento en mindfulness en la potenciación de la relajación y la autoeficacia escolar. Por lo tanto, el objetivo de este estudio fue comprobar si el entrenamiento en conciencia plena produce mejoras significativas en las variables de autoeficacia en el rendimiento escolar y en los estados de relajación (Estados-R), en una muestra de estudiantes de bachillerato.

\section{Método}

\section{Participantes}

En la presente investigación, participó un total de 43 estudiantes que cursaban primer y segundo curso de bachillerato. El $51 \%$ de los participantes eran chicas y el $49 \%$ chicos, cuyas edades oscilaban entre los 16 y los 19 años de edad $(M=17.05 ; D E=0.78)$. El grupo control estuvo formado por 22 sujetos ( $55 \%$ chicas y $45 \%$ chicos), mientras que los 21 sujetos restantes formaron parte del grupo experimental (47\% chicas y 53 \% chicos). La asignación a uno u otro grupo se realizó de manera aleatoria (Inglés et al., 2014; Gastaldi, F. G. M., Pasta, T., Longobardi, C., Prino, L. E., \& Quaglia, R., 2014), controlándose la variable sexo para que hubiera un número similar de chicos y chicas en ambos grupos, y así evitar la interferencia de esta variable en los resultados del estudio.

\section{Diseño}

Para analizar los efectos del programa de mindfulness (variable independiente) sobre los estados de relajación y sobre la autoeficacia en el rendimiento escolar (variables dependientes) se utilizó un diseño experimental de comparación de grupos, con medición pretest/postest, con un grupo experimental y un grupo control en lista de espera. 


\section{Instrumentos}

- Smith Relaxation States Inventory 3 ([SRSI-3]; Smith, 2005a) es un inventario que consta de 38 ítems tipo Likert en una escala de 1-6. La fiabilidad (alpha Chronbach) es de 0.88. Existen dos versiones: la versión "Estado" que mide los estados de relajación experimentados en el momento presente. Esta versión resulta apropiada para medir los efectos de una sesión de relajación después del entrenamiento. Por su parte, la versión "Rasgo" mide la propensión de cada individuo a experimentar los Estados-R a lo largo del tiempo. El cuestionario se compone de las siguientes escalas: Relajación Básica (integrada por Estados- $\mathrm{R}$ asociados a la relajación física, el descanso y la renovación, y la relajación mental), Energía Positiva (contiene Estados-R relacionados con energía y afecto positivo como por ejemplo, feliz, optimista...), Mindfulness (Estados$\mathrm{R}$ de silencio mental, consciencia y aceptación) y Transcendencia (Estados-R de Asombro, Devoción o Reverencia, Atemporalidad...). Adicionalmente, el cuestionario mide el Estrés percibido (Estrés Somático, Preocupación y Emociones Negativas).

- Cuestionario de Cartagena (Cartagena, 2008). La variable autoeficacia en el rendimiento escolar de los adolescentes fue evaluada con este cuestionario, mediante 20 ítems tipo Likert de 0 a10 puntos. Respecto a la validez y la fiabilidad de la prueba, el coeficiente alpha de Cronbach arroja una puntuación de 0.91 , siendo su fiabilidad de 0.88 .

\section{Procedimiento}

En primer lugar, se procedió a la selección de los sujetos participantes en la investigación. Para ello, se contactaron los directores de los centros participantes en el estudio, con el fin de ofertar una actividad extraescolar titulada "Aprendizaje y práctica de la conciencia plena en el contexto escolar", dirigida a los alumnos que se encontraban cursando primer y segundo curso de bachillerato. Al curso se inscribieron un total de 46 estudiantes, de los cuales 43 pasaron a formar parte de la investigación, ya que no se tuvieron en cuenta para los resultados del estudio a aquellos estudiantes que manifestaron haber tenido experiencia con alguna técnica de relajación, meditación, yoga, taichi, etc. A continuación, y antes de la asignación aleatoria de los participantes a cada uno de los grupos, se procedió a la evaluación pretest de los distintos componentes de los estados de relajación así como de los niveles de autoeficacia en el rendimiento escolar de cada uno de los participantes, mediante la aplicación de los cuestionarios descritos en el apartado anterior.

Una vez obtenida dicha medición inicial, y realizada la asignación a cada uno de los grupos, se procedió a la aplicación en el grupo experimental del programa de intervención, el cual consistía en el aprendizaje de una técnica de mindfulness para el entrenamiento y desarrollo de la conciencia plena denominada "Meditación Fluir" (Franco, 2009c). Mientras tanto, a los sujetos del grupo control se les indicó que con ellos se iniciaría dicho programa en el tercer trimestre del curso escolar.

El programa de intervención aplicado tuvo una duración de 8 semanas (segundo trimestre del curso académico), con una periodicidad de una sesión semanal de entre 1:30 y 2 horas de duración cada una de ellas. La técnica de mindfulness consiste en la repetición de una palabra carente de significado con una actitud mental libre y abierta, mientras se dirige la atención a la zona del abdomen para notar cómo entra y sale el aire de la respiración, pero sin tratar de modificar o alterar dicho proceso respiratorio, ya que tan solo hay que ser conscientes de cómo ocurre dicho proceso de forma natural y sin esfuerzo.

A los sujetos se les explicó que el objetivo de esta técnica no es tratar que la mente deje de pensar y de generar pensamientos, ni controlar los pensamientos, ni modificarlos o cambiarlos por otros, sino el desarrollar un estado de atención y conciencia plena sobre dicha actividad mental, siendo conscientes de la transitoriedad e impermanencia de dicha actividad, y aceptando cualquier pensamiento que pueda aparecer o surgir de forma espontánea. De este modo, lo que es esencial cuando se practica la conciencia plena no son los pensamientos en sí mismos, sino el hecho de ser conscientes de ellos sin evaluarlos, juzgarlos, ni analizarlos, simplemente viendo 
como aparecen y desaparecen, mientras se van dejando pasar. Adicionalmente, se realizaron ejercicios de exploración corporal o body-scan (Kabat-Zinn, 2003), que es una técnica donde la atención es puesta sobre diferentes partes del cuerpo de forma ordenada y sistemática, expandiendo posteriormente la conciencia a la totalidad del mismo, siendo conscientes de él de forma holística, como un todo; y todo ello, sin realizar ningún tipo de juicio o valoración, y sin intentar cambiar o eliminar nada (sensaciones corporales, reacciones mentales, sentimientos), estando presentes, momento a momento.

Los cuatro componentes principales que se trabajan a lo largo de las diferentes sesiones que componen el programa de intervención son: la aceptación, el perdón, la gratitud y la compasión. Este programa de entrenamiento es completado con diversas metáforas y ejercicios donde se comprende de forma experiencial que los pensamientos surgen y desaparecen continuamente y que están sometidos a un continuo flujo, aprendiendo, de esta forma, a estar presentes, abiertos y equilibrados frente a cualquier fenómeno o proceso mental o emocional que pueda acontecer.

Para facilitar la implementación del programa, los sujetos tenían que realizar diariamente en casa el ejercicio de body-scan durante 10 minutos y la práctica de conciencia plena sobre la respiración en la zona del abdomen durante 20 minutos. Además, cada participante debía completar a lo largo de la semana una hoja de autorregistro en la que diariamente debía anotar si había practicado los ejercicios de body-scan y de conciencia plena en la respiración, prescritos para su realización en casa. El grado medio de cumplimiento del ejercicio de body-scan fue del $71 \%$, mientras que del ejercicio de conciencia plena sobre la respiración repitiendo una palabra fue del $82 \%$. No se tuvieron en cuenta para los resultados del estudio a aquellos participantes que acudieron a menos del $50 \%$ de las sesiones del programa de intervención o que en la hoja de autorregistro informaron haber practicado menos del $50 \%$ de los días el ejercicio de body-scan y/o el ejercicio de conciencia plena. Dos participantes no cumplieron con dichos requisitos, por lo que sus puntuaciones no fueron consideradas para el análisis de los resultados.

Al finalizar el programa psicoeducativo de mindfulness en el grupo experimental, se procedió a evaluar nuevamente los niveles de los distintos componentes de los estados de relajación así como de los niveles de autoeficacia en el rendimiento escolar de los sujetos de los grupos control y experimental, en las mismas condiciones y con los mismos instrumentos que los empleados antes de la intervención, con la finalidad de comprobar, de este modo, si se habían producido modificaciones significativas en las variables objeto del estudio.

Todos los sujetos participantes en la investigación fueron informados a la finalización de la misma del objetivo del estudio llevado a cabo, y se les solicitó su consentimiento por escrito para poder hacer uso de los datos obtenidos manteniendo y garantizando la confidencialidad y el anonimato. Una vez finalizada la investigación, se impartió el programa de mindfulness a los sujetos del grupo control.

\section{Resultados}

Para la realización de los análisis del presente estudio se utilizó el paquete estadístico SPSS versión 21.0. La Tabla 1 muestra las medias y desviaciones típicas pretest y postest correspondientes a los grupos control y experimental para la variable autoeficacia en el rendimiento escolar y para las dimensiones del cuestionario de estados de relajación (SRSI-3).

Para comprobar la existencia de diferencias estadísticamente significativas entre las puntuaciones medias del grupo control y el experimental en cada una de las fases del estudio para la variable autoeficacia en el rendimiento escolar y para las dimensiones del cuestionario de estados de relajación, se empleó el estadístico de contraste no paramétrico $U$ de Man-Whitney para muestras independientes, ya que los datos no se ajustaban a la distribución normal de probabilidades. Al realizar dicho análisis estadístico para las puntuaciones pretest, se observa como no hay diferencias significativas de partida entre las puntaciones medias del grupo control y experimental en las variables del estudio. 
TABLA 1

Medias y desviaciones típicas pretest y postest correspondientes a los grupos control y experimental para la variable autoeficacia en el rendimiento escolar y para las dimensiones del cuestionario de estados de relajación

\begin{tabular}{lcccccccc}
\hline & \multicolumn{4}{c}{ PRETEST } & \multicolumn{4}{c}{ POSTEST } \\
\hline & \multicolumn{2}{c}{ Control } & \multicolumn{2}{c}{ Experimental } & \multicolumn{2}{c}{ Control } & \multicolumn{2}{c}{ Experimental } \\
\hline Variable & $M$ & $D E$ & $M$ & $D E$ & $M$ & $D E$ & $M$ & $D E$ \\
Autoeficacia & 47.7 & 12.1 & 44.8 & 11 & 48.9 & 12.8 & 56.3 & 9.8 \\
Relajación Básica & 25.5 & 7.4 & 23.9 & 6.1 & 26 & 8.2 & 31.6 & 5.4 \\
Conciencia Plena & 19.9 & 4.6 & 19.2 & 3.6 & 19.7 & 4.8 & 25.2 & 4 \\
Energía Positiva & 15.9 & 3.6 & 14.9 & 2.8 & 17.1 & 3.1 & 20.5 & 3.3 \\
Transcendencia & 9.9 & 2.6 & 9.6 & 2.3 & 10.3 & 3 & 10.8 & 2.4 \\
Estrés & 22.6 & 5.7 & 23.1 & 4.9 & 21.8 & 5.6 & 17.6 & 4.5 \\
\hline
\end{tabular}

Fuente: elaboración propia

Pero por el contrario, sí aparecieron diferencias estadísticamente significativas entre el grupo control y el experimental al realizar la diferencia de medias entre sus puntuaciones postest en la variable autoeficacia en el rendimiento escolar, así como en todas las dimensiones del cuestionario de estados de relajación excepto en la dimensión de trascendencia (Tabla 2).

Posteriormente, para comprobar la existencia de diferencias significativas entre las puntuaciones medidas pretest/postest de la variable autoeficacia en el rendimiento escolar y para las dimensiones del cuestionario de estados de relajación tanto en el grupo control como experimental, se empleó el estadístico de contraste no paramétrico de Wilcoxon. De este modo, al realizar dicho análisis entre las puntuaciones del grupo experimental, se obtuvieron diferencias significativas al comparar sus puntuaciones pretest/postest en la variable autoeficacia en el rendimiento escolar y en todas las dimensiones del cuestionario de estados de relajación. Sin embargo, al realizar dicha prueba para muestras relacionadas para las variables estudiadas en el grupo control, no se obtuvieron diferencias significativas en dichas variables al realizar las comparaciones de las puntuaciones medias pretest/ postest (Tabla 3).

Con el objetivo de poder valorar la magnitud del cambio ocurrido entre las puntuaciones pretest/postest en los grupos control y experimental, se empleó la $d$ de Cohen (1988). Valores superiores a 1.5 indi- can cambios muy importantes, entre 1.5 y 1 cambios importantes y entre 1 y 0.5 , cambios medios. En la Tabla 3 se observa como las puntuaciones de la $d$ de Cohen entre las medidas pretest/postest en el grupo experimental, muestran la existencia de cambios muy importantes en energía positiva y en conciencia plena; cambios importantes en relajación básica, estrés y autoeficacia escolar; apareciendo cambios de nivel medio en trascendencia. Por su parte, las puntuaciones de la $d$ de Cohen en el grupo control muestran la existencia de cambios bajos y muy bajos en todas las variables del estudio (Tabla 3).

Finalmente, se calculó el porcentaje de cambio entre las puntuaciones pretest/postest, observándose en el grupo experimental incrementos entre el 25 y el $37 \%$ en autoeficacia en el rendimiento escolar, relajación básica, conciencia plena y energía positiva, así como una reducción del $24 \%$ en estrés (Tabla 3). Sin embargo, los porcentajes de cambio en el grupo control oscilan entre el $7 \%$ en energía positiva y el $1 \%$ en conciencia plena.

\section{Discusión}

Los resultados obtenidos confirman que el entrenamiento en el programa psicoeducativo basado en técnicas mindfulness (conciencia plena) resulta efectivo en estudiantes de bachillerato, para incrementar la autoeficacia en el rendimiento escolar y potenciar los estados de relajación. Las medidas de evaluación utilizadas demostraron que como 


\section{TABLA 2}

Prueba U de Man-Whitney para muestras independientes de las diferencias pretest y postest entre el grupo control y experimental para la variable autoeficacia en el rendimiento escolar y para las dimensiones del cuestionario de estados de relajación

\begin{tabular}{lcccc}
\hline & \multicolumn{2}{c}{ PRESTEST } & \multicolumn{2}{c}{ POSTEST } \\
\hline Variable & $z$ & $p$ & $z$ & $p$ \\
Autoeficacia & 0.775 & 0.443 & 2.04 & $0.048^{*}$ \\
Relajación Básica & 0.739 & 0.464 & 2.53 & $0.015^{*}$ \\
Conciencia Plena & 0.53 & 0.599 & 3.88 & $0 * * *$ \\
Energía Positiva & 1.05 & 0.3 & 3.16 & $0.003^{* *}$ \\
Transcendencia & 0.38 & 0.706 & 0.571 & 0.571 \\
Estrés & 0.326 & 0.746 & -3.1 & $0.004^{* *}$ \\
\hline
\end{tabular}

Nota. $* * * p<0.0001 ; * * p<0.01 ;{ }^{*} p<0.05$.

Fuente: elaboración propia

TABLA 3

Prueba de Wilcoxon para muestras relacionadas de las diferencias postest-pretest y d de Cohen y porcentaje de cambio (\%) en el grupo control y experimental para la variable autoeficacia en el rendimiento escolar y para las dimensiones del cuestionario de estados de relajación

\begin{tabular}{lcccccccc}
\hline & \multicolumn{4}{c}{ Control } & \multicolumn{4}{c}{ Experimental } \\
\hline Variable & $z$ & $p$ & $d$ & $\%$ & $z$ & $p$ & $d$ & $\%$ \\
Autoeficacia & 1.59 & 0.126 & 0.09 & 2.55 & 8.77 & 0 & 1.1 & 25.6 \\
Relajación Básica & 1.07 & 0.298 & 0.06 & 1.96 & 7.76 & $0 * *$ & 1.34 & 32.2 \\
Conciencia Plena & 0.592 & 0.561 & 0.04 & 1.05 & 7.17 & $0^{* *}$ & 1.58 & 31.2 \\
Energía Positiva & 1.41 & 0.138 & 0.35 & 7.54 & 7.85 & $0^{* *}$ & 1.83 & 37.5 \\
Transcendencia & 1.75 & 0.095 & 0.14 & 4.04 & 3.14 & $0.003 *$ & 0.51 & 12.5 \\
Estrés & 0.102 & 0.92 & 0.15 & 3.53 & -5.92 & $0^{* *}$ & 1.17 & -24.3 \\
\hline
\end{tabular}

Nota: $* * p<0.001 ; p<0.01$.

Fuente: elaboración propia

consecuencia de la intervención los estudiantes entrenados obtuvieron índices más altos de autoeficacia en el rendimiento escolar y en las dimensiones del cuestionario de estados de relajación en todas sus escalas excepto la de Transcendencia. Este resultado está en línea con lo esperado, ya que el desarrollo de un estado de transcendencia en sí mismo requiere un tiempo de entrenamiento mayor de ocho semanas. Paralelamente, el grupo experimental obtuvo un descenso significativo en la escala de estrés percibido, estando este resultado en concordancia con los encontrados en el estudio de Franco (2009d), donde también se obtuvo una reducción significativa de los niveles de estrés percibido en estudiantes universitarios. En contraste, el grupo control no obtuvo aumentos ni descensos significativos en ninguna de las variables citadas, a pesar de que, antes de iniciarse el tratamiento, este grupo presentaba puntuaciones más altas en las variables de autoeficacia y relajación y menores en estrés percibido, si bien estas diferencias no eran significativas.

Diversas investigaciones han puesto de manifiesto cómo niveles altos de estrés y ansiedad producen alteraciones del triple sistema de respuestas implicado en el rendimiento académico (cognitivo, motor y fisiológico), produciendo una disminución del mismo. Las consecuencias incluyen déficits de atención y concentración, dificultades para memorizar y resolver problemas, déficits en las habilidades de estudio, escasa productividad y un bajo rendimiento académico (López, 2007; Maldonado, Hi- 
dalgo, \& Otero, 2000; Pérez, Martín, Borda, \& Del Río, 2003). En este sentido, los estudiantes con alto nivel de ansiedad tienden a focalizar su atención en la dificultad de la tarea, así como en sus fracasos académicos y en su falta de habilidades personales.

Durante la práctica de mindfulness, la mente está a la vez relajada y atenta. Se produce un estado fisiológico de profunda relajación, con una reducción del ritmo metabólico, cardiaco y respiratorio, mientras se mantiene un estado mental plenamente despierto y particularmente alerta. Este estado hipometabólico es totalmente opuesto al estado de alarma-defensa característico de la reacción de escape o lucha que desencadena una situación de estrés (Jevning, 1988; Smith, 2005b). Mediante la práctica continuada, los estudiantes pueden aprender a mantener la mente calmada y a concentrarse en el momento presente, llegando a experimentar la subjetividad y transitoriedad de sus pensamientos y sentimientos y pudiendo distanciarse de estos y observarlos (metacognición) desde una nueva perspectiva que facilita la reflexión y el aprendizaje (Grabovac, Lau, \& Willett, 2011). De esta forma, aprenderán a prevenir pensamientos rumiativos y obsesivos sobre acontecimientos pasados y futuros, que pueden estar relacionados con el estrés, o con pensamientos de ineficacia, que dificultan la capacidad de concentración del estudiante en su actividad académica.

De forma complementaria, hay que destacar los efectos beneficiosos de la experiencia de los estados de relajación (Estados-R) asociados a los componentes de relajación (Relajación Básica, Energía, Conciencia) y su efecto positivo en el aprendizaje (Amutio, 2002a, 2002b; Smith, 2005). Se sabe que las habilidades emocionales contribuyen a la adaptación y rendimiento académico del estudiante. Al mismo tiempo, el poner en práctica una habilidad provoca emociones positivas, incluida una mayor motivación. El trabajo en la escuela y el desarrollo intelectual requiere la habilidad de usar y regular emociones para facilitar el pensamiento, aumentar la concentración, desarrollar la motivación intrínseca, controlar el pensamiento impulsivo y actuar de forma efectiva bajo estrés. Así, la experiencia de los estados emocionales asociados a la relajación/ mindfulness puede provocar una reestructuración cognitiva de las creencias de ineficacia o incompetencia (Amutio, 2011; Amutio \& Smith, 2008).

Estos hallazgos están en línea con los resultados obtenidos en otras investigaciones en poblaciones no clínicas, donde la práctica del mindfulness ha sido asociada a una mayor relajación, bienestar y competencia escolar (Gilar, Miñano, \& Castejón, 2008; Schonert-Reichl \& Lawlor, 2010). Los resultados obtenidos resultan relevantes teniendo en cuenta los altos niveles de fracaso académico en los niveles de la Educación Secundaria Obligatoria y el bachillerato en España, que rondan alrededor del 25-30\%, según datos reportados en 2011 por el Ministerio de Educación y Ciencia. La alta tasa de fracaso escolar existente junto con la alta tasa de prevalencia de estrés y ansiedad entre estudiantes de bachillerato y universitarios (Amutio \& Smith, 2008; López, 2007) hace necesaria la puesta en marcha de estos programas psicoeducativos, con el objetivo de activar los recursos internos de los estudiantes, incluida la autoeficacia. Por su parte, esta última puede funcionar como amortiguador ante los estresores, haciendo que estos tengan un impacto menos negativo en el individuo y contribuyendo a que este adopte estrategias de afrontamiento adecuadas y obtenga un mejor rendimiento.

En definitiva, el mindfulness y la relajación constituyen estrategias de afrontamiento altamente efectivas para manejar las emociones negativas, incrementar el bienestar y mejorar el rendimiento. Por este motivo, resulta muy importante el aprendizaje y cultivo de las habilidades de relajación y mindfulness en la adolescencia a través de la enseñanza de prácticas meditativas formales (escaneo corporal, estiramientos de yoga, meditación sentada) y la integración de esta capacidad en la vida diaria como un recurso de afrontamiento de los síntomas físicos y emocionales. Todo esto implica también la necesidad de proporcionar una formación especializada al profesorado en técnicas de mindfulness. De este modo, los estudiantes de bachillerato tendrán un mayor conocimiento de sí mismos y aprenderán a manejar con mayor efectividad las diversas situaciones de estrés que deben afrontar durante este período, sabiendo desenvolverse de forma eficaz 
en un mundo cada vez más flexible y cambiante. La adquisición de estas habilidades forma parte del desarrollo integral de los alumnos de bachillerato, a fin de prevenir la aparición de emociones como la ansiedad y la depresión y sus negativas consecuencias sobre el rendimiento. Todo esto implica también la necesidad de proporcionar una formación especializada al profesorado en técnicas mindfulness.

Uno de los puntos fuertes de este estudio es su novedad, ya que se utiliza una conceptualización amplia de la relajación como un proceso psicológico que tiene unos efectos concretos a través de la experiencia de diversos estados emocionales altamente positivos, superándose el reduccionismo de los modelos biomédicos existentes. Además, se trata de un estudio con grupo control aleatorizado y con una alta tasa de adherencia al programa. Sin embargo, el presente trabajo presenta algunas limitaciones. En primer lugar, existe la posibilidad de que se haya producido un sesgo de autoselección de la muestra. En segundo lugar, porque la muestra del estudio es relativamente pequeña. Por ello, se ha de ser cautos en cuanto a la validez externa o generalización de los resultados, y se requiere de estudios que repliquen los hallazgos obtenidos utilizando muestras más amplias. Otro aspecto importante que se debe tener en cuenta es la carencia de tratamiento alternativo para el grupo control, lo que impide la neutralización de los efectos de demanda y placebo en el grupo experimental. Por último, sería importante llevar a cabo un seguimiento más allá de las ocho semanas para comprobar la duración y o el mantenimiento de los cambios. Estudios futuros que serán realizados por nuestro equipo de investigación podrán subsanar las limitaciones mencionadas.

En resumen, y a pesar de dichas limitaciones, enfatizamos la importancia del programa psicoeducativo propuesto en formato grupal, en cuanto que supone una importante optimización de los recursos económicos y sociales invertidos en la educación.

\section{Referencias}

Amutio, A. (junio, 2013). Mindfulness y bienestar: resultados de un estudio longitudinal. Libro de Resúme- nes del VI Congreso Internacional y XI Nacional de Psicología Clínica, Santiago de Compostela, Galicia, España.

Amutio, A. (2011). Autorregulación emocional y control del estrés: relajación y reevaluación cognitiva. En M. Álvarez \& R. Bisquerra (Coords.), Manual de orientación y tutoría [CD] (pp. 1-15). Madrid: Wolters-Kluwer.

Amutio, A., \& Smith, J. C. (2008). Stress and irrational beliefs in college students. Ansiedad y Estrés, 14(2-3), 211-220.

Amutio, A. (2002a). Relajación y emociones positivas. Ansiedad y Estrés, 8(1), 59-71.

Amutio, A. (2002b). Estrategias de manejo del estrés: el papel de la relajación. Cuadernos de Medicina Psicosomática y Psiquiatría de Enlace, 62-63, 19-31.

Bandura, A. (1997). Self-efficacy: The exercise of control. Englewood Cliffs, NJ: Prentice Hall.

Beaucheim, J. Hutchins, T., \& Patterson, F. (2008). Mindfulness meditation may lessen anxiety, promote social skills, and improve academic performance among adolescents with learning disabilities. Complementary Health Practice Review, 3(1), 34-35.

Bishop, S. R., Lau, M., Shapiro, S., Carlson, L. E., Anderson, N., \& Carmody, J. (2004). Mindfulness: A proposed operational definition. Clinical Psychology: Science and Practice, 11(3), 230-241.

Brefczynski-Lewis, J. A., Lutz, A., Schaefer, H. S., Levinson, D. B., \& Davidson, R. J. (2007). Neural correlates of attentional expertise in long-term meditation practitioners. Proceedings of the $\mathrm{Na}$ tional Academy of Sciences of the United States of America, 104(27), 11483-11488.

Calvo, F., Betancort, E., \& Díaz, M. D. (2009). La técnica de relajación sugestiva breve automatizada: ampliación del estudio de su eficacia a una muestra de universitarios. Ansiedad y Estrés, 15(2-3), 119-130.

Cartagena, M. (2008). Relación entre la autoeficacia y el rendimiento escolar y los hábitos de estudio en alumnos de secundaria. Revista Iberoamericana sobre Calidad Eficacia y Cambio en Educación, 6(3), 59-99.

Cebolla, A., \& Miró, M. T. (2006). Eficacia de la terapia cognitiva basada en la atención plena en el tratamiento de la depresión. Revista de Psicoterapia, 17(66-67), 133-155. 
Chiesa, A., \& Serretti, A. (2009). Mindfulness-based stress reduction for stress management in healthy people: A review and meta-analysis. The Journal of Alternative and Complementary Medicine, 15(5), 593-600.

Choi, Y., Karremans, J. C., \& Barendregt, H. (2012). The happy face of mindfulness meditation is associated with perceptions of happiness as rated by outside observers. The Journal of Positive Psychology, 7(1), 30-35.

Davidson, R. J., Kabat-Zinn, J., Schumacher, J., Roserkranz, M. S., Muller, D., Santorelli, S. F., ... Sheridan, J. F. (2003). Alterations in brain and immune function produced by mindfulness. Psychosomatic Medicine, 65(4), 564-570.

Delgado, L. C., \& Amutio, A. (2011). La ansiedad generalizada y su tratamiento basado en mindfulness. Cuadernos de Medicina Psicosomática y Psiquiatría de Enlace, 99, 50-65.

Delgado, L. C., Guerra, P., Perakakis, P., Viedma, M. I., Robles, H., \& Vila, J. (2010). Eficacia de un programa de entrenamiento en conciencia plena (mindfulness) y valores humanos como herramienta de regulación emocional y prevención del estrés para profesores. Behavioral Psychology/Psicología Conductual, 18(3), 511-532.

Franco, C. (2009b). Reducción de la percepción del estrés en estudiantes de Magisterio mediante la práctica de la meditación fluir. Apuntes de Psicología, 27(1), 99-109.

Franco, C. (2009c). Meditación fluir para serenar el cuerpo y la mente. Madrid: Bubok.

Franco, C. (2009d). Reducción de la percepción del estrés en estudiantes de Magisterio mediante la práctica de la meditación fluir. Apuntes de Psicología, 27(1), 99-109.

Franco, C. (2010a). Reducing psychological distress in inmigrants living in Spain thorugh the practice of flow meditation. European Journal of Education and Psychology, 2(3), 223-233.

Franco, C. (2010b). Intervención sobre los niveles de burnout y resiliencia en docentes de educación secundaria a través de un programa de conciencia plena (mindfulness). Revista Complutense de Educación, 21(2), 271-288.
Franco, C., Mañas, I., Cangas, A. J., \& Gallego, J. (2011). Exploring the effects of a mindfulness program for students of secondary school. International Journal of Knowledge Society Research, 2(1), 14-28.

Gastaldi, F. G. M., Pasta, T., Longobardi, C., Prino, L. E., \& Quaglia, R. (2014). Measuring the influence of stress and burnout in teacher-child relationship. European Journal of Education and Psychology, 7(1), 17-28.

Gilar, R., Miñano, P., \& Castejón, J. L. (2008). Inteligencia emocional y empatía: su influencia en la competencia social en Educación Secundaria Obligatoria. SUMMA Psicológica UST, 5(1), 21-32.

Grabovac, A. D., Lau, M. A., \& Willett, B. R. (2011). Mechanisms of mindfulness: A Buddhist psychological model. Mindfulness, 2(3), 154-166. doi: 10.1007/s12671-011-0054-5

Inglés, C. J., Torregrosa, M. S., García-Fernández, J. M., Martínez-Monteagudo, M. C., Estévez, E., \& Delgado, B. (2014). Conducta agresiva e inteligencia emocional en la adolescencia. European Journal of Education and Psychology, 7(1), 29-41.

Jevning, R. (1988). Integrated metabolic regulation during states of decreased metabolism, similarity to fasting: A biochemical hypothesis. Physiology and Behavior, 43, 735-737.

Kabat-Zinn, J. (1982). An outpatient program in behavioral medicine for chronic pain patients based on the practice of mindfulness meditation. General Hospital Psychiatry, 4, 33-47.

Kabat-Zinn, J. (2003). Vivir con plenitud las crisis. Cómo utilizar la sabiduría del cuerpo y de la mente para afrontar el estrés, el dolor y la enfermedad. Barcelona: Kairós.

Kaspereen, D. (2012). Relaxation intervention for stress reduction among teachers and students. International Journal of Stress Management, 19(3), 238-250.

León, B. (2008). Atención plena y rendimiento académico en estudiantes de enseñanza secundaria. European Journal of Education and Psychology, 1(3), $17-26$.

López, L. (2007). Relajación en el aula: recursos para la educación emocional. Madrid: Wolters Kluwer.

Maldonado, M. D., Hidalgo, M. J., \& Otero, M. D. (2000). Programa de intervención cognitivo-conductual y de técnicas de relajación como método 
para prevenir la ansiedad y el estrés en alumnos universitarios de enfermería y mejorar el rendimiento académico. Cuadernos de Medicina Psicosomática y Psiquiatría de Enlace, 53, 43-57.

Mañas, I., Sánchez, L. C., \& Luciano, M. C. (abril, 2008). Efectos producidos por un ejercicio de mindfulness (body-scan): un estudio piloto. Póster presentado en el VII Congreso de la Sociedad Española de Psicología Experimental, San Sebastián-Donostia, España.

Martín-Asuero, A., Rodríguez, T., Pujol-Ribera, E., Berenguera, A., \& Moix, J. (2013). Evaluación de un programa de mindfulness en profesionales de atención primaria. Gaceta Sanitaria, 27(6), 521-528.

Ministerio de Educación y Ciencia. (2011). Datos y cifras curso escolar 2011/2012. Madrid: Secretaría General Técnica. Subdirección General de Documentación y Publicaciones, Catálogo General de Publicaciones Oficiales.

Pérez, M. A., Martín, A., Borda, M., \& Del Río, C. (2003). Estrés y rendimiento académico en estudiantes universitarios. Cuadernos de Medicina Psicosomática y Psiquiatría de Enlace, 67-68, 26-33. Santamaría, M. T., Cebolla, A., Rodríguez, P. J., \& Miró, M. (2006). La práctica de la meditación y la aten- ción plena: técnicas milenarias para padres del Siglo XXI. Revista de Psicoterapia, 17(66-67), 157-175.

Schonert-Reichl, K. A., \& Lawlor, M. S. (2010). The effects of a mindfulness-based education program on pre- and early adolescents' well-being and social and emotional competence. Mindfulness, 1(3), 137-151. doi: 10.1007/s12671-010-0011-8

Simón, V. (2006). Mindfulness y neurobiología. Revista de Psicoterapia, 17(66-67), 5-31.

Smith, J. C. (2005a). Smith Relaxation States Inventory

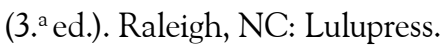

Smith, J. C. (2005b). Relaxation, meditation y mindfulness: A mental health practitioner's guide to new and traditional approaches. New York: Springer.

Vago, D. R., \& Silbersweig, D. A. (2012). Self-awareness, self-regulation, and self-transcendence (S-ART): Framework for understanding the neurobiological mechanisms of mindfulness. Frontiers in Human Neuroscience, 6(1), 1-30.

Vallejo, M. A. (2006). Mindfulness (presencia reflexiva y atenta). Psicología Conductual, 14(3), 433-451.

Wells, A. (2002). Metacognition and mindfulness: An information processing analysis. Clinical Psychology Science and Practice, 9(1), 95-100. 
